\title{
Patient experience following iliac crest- derived alveolar bone grafting and implant placement
}

\author{
Cecilie G. Gjerde ${ }^{1,2^{*}} \mathbb{D}$, Siddharth Shanbhag ${ }^{2}$, Evelyn Neppelberg ${ }^{1,3}$, Kamal Mustafa ${ }^{2}$ and Harald Gjengedal ${ }^{4}$
}

\begin{abstract}
Background: The objective of this study was to assess patient-reported outcomes such as satisfaction and quality of life after advanced alveolar bone augmentation with anterior iliac crest grafting and implant treatment in orally compromised patients.
\end{abstract}

Methods: This cross-sectional retrospective cohort study included 59 patients (29 women and 30 men) with major functional problems, who underwent advanced alveolar augmentation with autologous iliac bone grafts during a 10-year period (2002-2012).

The self-administered questionnaire included 36 validated questions related to (1) demographics, (2) perceived general and oral health, (3) donor site and hospitalization, (4) status of implants and/or prosthesis, and (5) oral health-related quality of life (OHRQoL).

Results: Questionnaires were completed by 44 patients: 24 women and 20 men (response rate, 74.6\%). Most patients reported good tolerance of the operative iliac bone harvesting (85\%) and implant (90\%) procedures. Postoperative pain at the donor site was reported by $38 \%$, lasting $18.1 \pm 16.1$ days. An average of $4.3 \pm 3.5$ days of hospitalization and $20.2 \pm 18.5$ days of sick leave was reported. The overall satisfaction with prosthetic reconstruction was 90.5\%. OHRQoL was reported with a mean Oral Health Impact Profile-14 (OHIP-14) score of 8.4.

Conclusion: Favorable OHRQoL and satisfaction were reported after advanced reconstruction of alveolar ridges with iliac crest-derived grafting and implants in severely compromised patients. However, this treatment requires substantial resources including hospitalization and sick leave.

Keywords: Dental implants, Reconstruction, Quality of life, Bone graft, lliac crest, Donor site morbidity, PROMs

\section{Background}

Insufficient alveolar bone volume, as a result of periodontal disease, trauma, congenital anomalies and/or resorption atrophy, often presents a clinical challenge for optimal placement of dental implants for prosthetic rehabilitation. In such cases, augmentation of alveolar bone, with either autologous bone, allogeneic, xenogeneic, or alloplastic biomaterials, is a prerequisite for placing implants in restoratively and esthetically acceptable positions.

\footnotetext{
* Correspondence: cecilie.gjerde@uib.no

1 Department of Oral and Maxillofacial Surgery, Institute of Clinical Dentistry, University of Bergen, Årstadveien 19, 5009 Bergen, Norway

${ }^{2}$ Centre for Clinical Dental Research, Institute of Clinical Dentistry, University of Bergen, Bergen, Norway

Full list of author information is available at the end of the article
}

Limited alveolar ridge defects are solved by local grafting. In cases of larger defects and extreme resorption, larger grafts are necessary. The most common donor site for large autologous bone grafts is the iliac crest, due to its accessibility, comparatively abundant bone volume, and high bone quality [1].

Autologous bone is still considered as a "gold standard" for alveolar reconstruction, according to systematic reviews [2-5]. Intra-oral donor sites, like mandibular ramus and symphysis, allow harvesting of limited volumes of autologous bone. The anterior iliac crest is the preferred extra-oral donor site for alveolar augmentation for larger bone volumes $[1,6,7]$. However, complications are reported, including pain, gait disturbance, hematomas, paranesthesia, and infections [8-15]. 
Traditionally, objective clinical variables, like the amount of bone gain (in millimeters) after augmentation, are reported as outcome measures after surgical procedures in clinical studies [16]. Patients' experiences like patient-reported outcome measures (PROMs) have been increasingly used as a measure of treatment effect after medical and dental therapies $[17,18]$. Importantly, these measures reflect the patients' perceptions of the treatment outcome in addition to conventional clinical measures. Nowadays, Norwegian authorities address clinicians to include patients' perspective in decisions regarding different treatment modalities [19]. It has been suggested that PROMs such as treatment satisfaction, perceived costeffectiveness, and quality of life (QoL) may be more important and relevant to patients' daily lives than objective clinical measures $[16,20]$. Patient satisfaction is an important outcome measure, related to, although not synonymous with QoL, as satisfaction tends to reflect the process, rather than the outcome, of care [21]. Thus, an increase in the use of PROMs has been highlighted in dental implant research [22].

Health-related QoL (HRQoL) is a dynamic concept referring to an individual's subjective assessment and perspective of current general health condition as well as functional, social, and emotional well-being [23, 24]. Most people regard oral health as important for QoL, and this is mediated through the concept of oral healthrelated QoL (OHRQoL) [25]. In this regard, OHRQoL is an important PROM in dental research, as oral health is an integral part of general health and well-being [26].

Different instruments to assess OHRQoL may be utilized to detect changes in physical, functional, and psychosocial impacts of oral disorders and have been validated for use in clinical studies [27-29]. The Oral Health Impact Profile-14 (OHIP-14) questionnaire is a widely used OHRQoL instrument [27]. It includes 14 questions covering seven domains of oral health and attempts to assess their impact on patients' OHRQoL [30, 31]. OHIP-14 has previously been translated into Norwegian and used in a large study $(n=3538)$ with a calculated Norwegian national norm value [32]. Although previous studies have reported PROMs in relation to bone grafting [9, 33-42], to our knowledge, only one previous study has systematically assessed impact of donor site harvesting on OHRQoL, where (a) a postoperative lowering of OHRQoL was observed following bone grafting from both intra-oral and extra-oral sites and (b) iliac crest grafts compared to intraoral donor sites had a negative impact on postoperative QoL [37]. Moreover, to our knowledge, only one study has previously assessed the cost-effectiveness of autologous iliac crest grafting [43].

The aim of this study was to assess PROMs such as satisfaction and OHRQoL after advanced reconstruction of alveolar bone by anterior iliac crest-derived grafting and implant treatment.

\section{Methods \\ Study population}

This cross-sectional retrospective cohort study was based on records from all patients $(n=69)$ who underwent advanced alveolar augmentation with autologous iliac bone grafts at the Department of Oral and Maxillofacial Surgery, Haukeland University Hospital, Bergen, Norway, over 10 years (2002-2012). These patients were orally compromised with severe chewing problems as well as speech difficulties and had previously undergone several unsuccessful rehabilitation methods, prior to referral. At the time of this survey, seven patients had passed away, two had moved to unknown addresses, and one was hospitalized in a psychiatric institution. Thus, the study sample included 59 patients: 29 women and 30 men.

The Norwegian Committee for Medical Research Ethics ("REK," Health Region West), acknowledged this study as a treatment quality control study.

\section{Treatment protocol-operative procedure}

Bone graft surgeries were performed under general anesthesia and sterile conditions. Cortico-cancellous bone blocks were harvested from the anterior superior iliac crest. Reconstructions in the maxilla $(N=57)$ or mandible $(N=2)$ were performed in one operation by two teams using an onlay bone graft fixated with titanium micro-screws $(1.5 \mathrm{~mm} \varnothing)$. The surgical procedure was performed according to the protocol commonly used at Haukeland University Hospital. In brief, the harvesting of autogenous bones from the anterior iliac crests started with a skin incision following the skin lines in a posterolateral direction starting from 3 to $4 \mathrm{~cm}$ medial to the iliac crests. The superior surfaces of the iliac crests are exposed after a sharp dissection through the periosteum following the crests. The dissections are performed with great attention to avoid laceration of the fascia lata. Both cortical and spongious bone are harvested. The donor sites are closed in layers with special attention to the first layer-the fascia lata. This layer is sutured close to avoid marrowbone bleeding. Activated vacuum drainages are positioned between the fascia lata and the muscles until the patients are mobilized. The skin incisions are closed with continuous intracutaneous resorbable sutures. All patients included in the study were hospitalized 2-3 days postoperatively. Patients received phenoxymethylpenicillin $(1 \mathrm{~g} \times 3)$ or clindamycin $(300 \mathrm{mg} \times 3)$ for 5 days following the operation. Vacuum drainage at the donor site was used until the patient was mobilized the morning after surgery. Analgesics (paracetamol or non-steroid anti-inflammatory drugs) were prescribed 7-10 days postoperatively. 
Implants were placed 4-6 months after the grafting procedure. The implant installations were performed by different oral surgeons (not in the hospital) and different implant systems were used. The implants installed into the augmented bone were allowed to heal for an additional 4-6 months before loading.

\section{Data collection}

\section{Medical records}

The records of the original 69 patients were examined with regard to (1) grafting site (2), "graft-survival" determined by the ability to place implants in the grafted site(s) and (3) "implant survival" determined by the presence of functional implant-supported prostheses at the most recent follow-up. Reasons for implant failure were recorded when available.

\section{Questionnaire}

A self-administered questionnaire (Additional file 1) was sent by post to all 59 patients, together with an information leaflet about the survey, a return envelope with prepaid postage and an informed consent form. Reminder letters were sent after 2 and 4 weeks if no response was received.

The questionnaire contained 36 previously validated questions, which were categorized and related to (1) demographic and lifestyle, (2) perceived general and oral health, (3) donor site and hospitalization, (4) implant and prosthesis, and (5) OHRQoL (OHIP-14) (Table 1). Responses to questions in categories 1-2 were recorded as "yes/no" or graded on a 3- to 5-point Likert scale [44]. Category 3 included information on the duration of hospitalization and sick leave. Category 4 included information on "graft survival," i.e., whether implants (and prostheses) were delivered in the augmented site(s), and "implant survival," i.e., the presence or "loss/loosening" of any implants after surgery. OHRQoL was assessed using a Norwegian version of the OHIP-14 [32]. These 14 questions addressed seven domains of OHRQoL and their responses were graded on a 5-point Likert scale ranging from "at no time" (0) to "all of the time" (4) (Table 1).

\section{Statistical analysis}

Data were anonymized and analyzed using SPSS v 24 (SPSS Inc., Chicago, IL, USA). Descriptive analyses were applied. Statistical significance was set at 5\% level.

\section{Results}

The final sample consisted of 44 patients that responded and completed the questionnaire, giving a response rate of $74.6 \%$ : 24 women and 20 men, mean age of 61.2 years \pm 13.1 (range $27-82$ years). The mean time from augmentation surgery until completing the questionnaire was 7.8 years \pm 2.65 (range $1.9-12$ years).
Table 1 Summary of questions

\begin{tabular}{ll}
\hline Category & Response \\
\hline Question & \\
(1) Perceived health-status & \\
General health & "Very good" to "bad" \\
Oral health & "Very good" to "bad" \\
Overall quality of life & "Excellent" to "bad" \\
(2) Lifestyle-related & "Yes," "no," or "sometimes" \\
Smoking & "Good" to "bad" \\
Appetite & \\
(3) Donor site-related & "Yes" and "no" \\
Pain & "Yes" and "no" \\
Infection & "Yes" and "no" \\
Presence of a scar & "No" to "total loss of \\
Reduced sensitivity & sensitivity" \\
Problems walking & "No" to "a lot" \\
Satisfaction & "Very satisfied" to "dissatisfied" \\
(4) Implant-related & "At no time" to "all of the \\
Intraoral pain & "No" to "strong pain" \\
Installation of implants and & "Yes", "no" or "just implants" \\
prosthetic & "Yoss of implants \\
Satisfaction with prosthesis & \\
(5) OHIP-14 & \\
\hline
\end{tabular}

Summary of demographic and lifestyle-related data is presented (Table 2).

\section{Descriptive findings \\ Health-related PROMs}

Most patients reported "good" or "very good" levels of general health $(81.4 \%)$, oral health $(83.7 \%)$, and overall quality of life (90.7\%). Less than 5\% reported "bad" levels for either of these variables. Most patients reported better general (86\%) and oral health (78\%) after treatment. Only two patients $(4.7 \%)$ reported their oral health to be worse after treatment.

\section{Donor site- and hospitalization-related PROMs}

Most patients (85.4\%) were satisfied with the hip surgery procedure. Pain at the donor site was reported by $38 \%$ of patients, lasting for an average of $18.1 \pm 16.1$ days and measuring $43.6 \pm 27$ on the VAS $(0-100)$ scale. Only two patients $(4.7 \%)$ reported post-operative infection at the donor site. Scar formation on skin (hip) was reported in $49 \%$ of patients, by majority esthetically acceptable (90.4\%). Four (9.5\%) and two (4.7\%) patients reported "a little" or "a lot" of reduced sensitivity at the donor site, respectively. Three patients (7.3\%) reported problems in 
Table 2 Patients' demographic and lifestyle-related data

\begin{tabular}{|c|c|c|}
\hline \multirow[t]{2}{*}{ Variable } & \multicolumn{2}{|l|}{ Frequency } \\
\hline & $\bar{N}$ or Mean $\pm S D$ & $\%$ \\
\hline \multicolumn{3}{|l|}{ Patients } \\
\hline Female & 24 & 54.5 \\
\hline Male & 20 & 45.5 \\
\hline Age (years) & $61.16 \pm 13.10$ & \\
\hline Age at operation & $53.73 \pm 13.07$ & \\
\hline $\begin{array}{l}\text { Time from augmentation to } \\
\text { completing questionnaire (months) }\end{array}$ & $93.55 \pm 31.75$ & \\
\hline \multicolumn{3}{|l|}{ Civil status } \\
\hline Married & 30 & 68.2 \\
\hline Single & 11 & 25.0 \\
\hline Widow(er) & 3 & 6.8 \\
\hline \multicolumn{3}{|l|}{ Housing } \\
\hline Alone & 12 & 27.3 \\
\hline With another person & 23 & 52.3 \\
\hline$>$ two persons & 9 & 20.5 \\
\hline \multicolumn{3}{|l|}{ Education } \\
\hline Up to primary & 7 & 11.3 \\
\hline Up to secondary & 23 & 53.5 \\
\hline "Artium" & 1 & 2.3 \\
\hline High school & 9 & 20.9 \\
\hline University & 3 & 7.0 \\
\hline \multicolumn{3}{|l|}{ Smoking } \\
\hline Yes & 8 & 19.0 \\
\hline No & 33 & 78.6 \\
\hline Sometimes & 1 & 2.4 \\
\hline Cigarettes/day & $13.65 \pm 7.22$ & \\
\hline Years of smoking & $26.52 \pm 11.63$ & \\
\hline
\end{tabular}

walking (Table 3). The average time of hospitalization was $4.3 \pm 3.5$ days and sick leave $20.2 \pm 18.5$ days.

\section{Implant-/prostheses-related PROMs}

Most patients $(n=40,90.9 \%)$ reported to have implants placed and received prostheses in the augmentation site(s). This was interpreted as graft survival, indicating a graft survival rate of $90.9 \%$ on the patient level. Two patients received implants, although without further prosthetic rehabilitation. Implants could not be installed in two patients. However, $29.3 \%$ of patients reported "loosening or loss" of implants in the post-operative period (1 year), indicating an implant survival rate on the patient level of $70.7 \%$, and most patients (8 out of 11) received new implants.

No pain was reported in 39 patients (82.9\%) following implant surgery and a majority of patients $(90.2 \%)$ were satisfied/very satisfied with the implant therapy overall and in terms of overall satisfaction with teeth (90.5\%).

The correlation analyses performed did not show a significant correlation between the complications at the donor site and implant loss (Table 4).

\section{OHRQoL}

The mean OHIP-14 score (Table 5) was $8.4 \pm 9.7$ (range 056 ) in 44 patients of whom 35 patients scored 14 or less. Nine patients scored a total sum of 1 [1], i.e. "hardly ever" impact on any single item and "at no time" on the remaining 13 items. The functional limitation domain had the highest score (2.34) and the social disability domain the lowest score (0.61).

\section{Discussion}

An important finding in this study is that a majority of patients were very satisfied after iliac crest-derived alveolar bone grafting and implant therapy. Although 90\% of the patients in our study had successful bone grafting, only $70.1 \%$ reported implant survival together with prosthetic rehabilitation after 1 year. These figures are lower than those reported in previous studies $[2,3,9]$. A review by Chiapasco et al. showed that the mean graft failure in 16 studies was $1.6 \%$ and partial loss of graft of $3.3 \%$ [45]. The same review showed that the overall survival rate of dental implants in transplanted bone was $87 \%$. However, it must be kept in mind that the patients in our study were orally compromised and very challenging to reconstruct. Moreover, the patients in our study did not report on the number of implants lost, and we do not have reliable records of the exact number of implants each patient had got installed. This could indicate differences in survival on implant or patient levels-a variable of clinical importance as the number of lost implants may be higher.

Another important finding is that patients reported to tolerate the augmentation procedure well; $85 \%$ of patients were satisfied with the hip operation (performed under general anesthesia), comparable to a previous report [46]. However, $40 \%$ of the patients reported pain for $18 \pm 16$ days after augmentation, which is in accordance with other studies [37, 46] and which should be considered during the treatment planning of patients scheduled to received iliac crest-derived bone grafts [33]. Two patients reported infection at the donor site. All operations were performed by a strict sterile regime and protocol at the university hospital.

The level of OHRQoL reported by the patients was favorable with an OHIP-14 value of 8.4. In a previous study, Dahl et al. reported an OHIP-14 score of 4.1 in the Norwegian adult population (2441 patients), with $35 \%$ of the sample reporting "no oral health problems" [32]. If the study sample in the study of Dahl et al. is considered to be representative of the general population, patients in our study reported poorer OHRQoL than the general population. Thus, even though the participants in this study report good oral health and better than before operation on the single questions, they 
Table 3 Patient-reported outcomes

\begin{tabular}{|c|c|c|}
\hline Question & Response & Frequency \\
\hline Oral health & Very good/good & $81.8 \%$ \\
\hline Quality of Life & Very good/good & $90.9 \%$ \\
\hline General health & Very good/good & $81.8 \%$ \\
\hline Pain after hip operation & Excessive & $35.0 \%$ \\
\hline Satisfaction hip operation & Very & $85.7 \%$ \\
\hline Post op infection in hip site & No & $95.3 \%$ \\
\hline Visible scar on hip & Yes & $48.8 \%$ \\
\hline Acceptable scar & Yes & 20 of $21^{a}$ \\
\hline Reduced sensibility on hip site & No & $86.0 \%$ \\
\hline Problem walking & No & $92.9 \%$ \\
\hline Augmented bone block still present & No & $6.8 \%$ \\
\hline New augmentation & Yes & 1 of $4^{a}$ \\
\hline Oral pain after augmentation & No/some & $83.3 \%$ \\
\hline Implant/teeth in augmented bone & Yes & $90.9 \%$ \\
\hline Lost implants & Yes & $28.6 \%$ \\
\hline \multirow[t]{2}{*}{ Time lost after installation } & $0-3$ months & $42.9 \%$ \\
\hline & 7-12 months & $28.6 \%$ \\
\hline New implants installed & Yes & 8 of $11^{a}$ \\
\hline Satisfaction with implant-retained teeth & Very satisfied/satisfied & $90.5 \%$ \\
\hline
\end{tabular}

Incomplete or missing data

still report having problems related to their oral condition. This is to be expected as the patients in our study were orally compromised before augmentation with almost no alveolar ridge to retain or support a prosthetic construction. Since the patients had extensive alveolar bone loss rendering them orally handicapped, any improvement in function would be likely to have a positive impact on satisfacation and OHRQoL. However, it is difficult to relate their reported level of

Table 4 Correlation analyses

\begin{tabular}{|c|c|c|c|}
\hline Outcome variables & Correlations & Spearman's rho & $P$ value \\
\hline \multirow[t]{8}{*}{ OHRQOL } & Oral health compared & 0.596 & $<0.0001$ \\
\hline & General health now & 0.369 & 0.014 \\
\hline & General health compared & 0.412 & 0.005 \\
\hline & Implants placed/teeth installed & 0.317 & 0.036 \\
\hline & Lost implants & -0.372 & 0.015 \\
\hline & Smoking & -0.334 & 0.005 \\
\hline & Speaking & 0.572 & $<0.0001$ \\
\hline & Chewing & 0.375 & 0.014 \\
\hline \multirow[t]{6}{*}{ Implants placed } & General health & -0.314 & 0.038 \\
\hline & Oral pain post op & 0.334 & 0.031 \\
\hline & Oral health & 0.305 & 0.044 \\
\hline & General health compared & 0.314 & 0.038 \\
\hline & Satisfaction hip operation & -0.439 & 0.004 \\
\hline & OHRQOL & 0.317 & 0.036 \\
\hline \multirow[t]{4}{*}{ Lost implants } & General health & -0.328 & 0.034 \\
\hline & QoL & -0.342 & 0.027 \\
\hline & OHRQOL & -0.372 & 0.015 \\
\hline & Satisfied teeth & -0.328 & 0.034 \\
\hline
\end{tabular}


Table 5 Summary of OHIP-14 ( $N=44$ and response range $0-8)$

\begin{tabular}{lllll}
\hline OHIP domain & Minimum & Maximum & Mean & SD \\
\hline Functional limitation & 0 & 7 & 2.34 & 1.70 \\
Physical pain & 0 & 7 & 1.16 & 1.51 \\
Psychological discomfort & 0 & 8 & 1.64 & 2.27 \\
Physical disability & 0 & 8 & 0.75 & 1.77 \\
Psychological disability & 0 & 8 & 1.18 & 2.11 \\
Social disability & 0 & 8 & 0.61 & 1.40 \\
Handicap & 0 & 8 & 0.70 & 1.71 \\
Total & 0 & 64 & 8.4 & 9.7 \\
\hline
\end{tabular}

OHRQoL to the augmentation and implant installation per se, as this was performed up to 12 years prior to completing the questionnaire (mean 7 years and 10 months). So, patients' present oral situation with fixed teeth could/may alter the "reference" for the patients regarding OHRQoL. However, we cannot reliably ascribe the level of OHRQoL to the treatment performed years ago, since we have no such data either before or soon after the prosthetic rehabilitation, and therefore, cannot estimate the influence the effect of response shift on the study outcomes. Previous reports show a significant influence of implantretained prosthetic treatment on OHRQoL, but these reports are based on before-and-after registrations [47].

Patients in our study reported satisfaction with the augmentation and implant installation, and as these patients were orally compromised before the operation, their satisfaction with getting fixed teeth most likely improved their perceived oral health condition. This might also, in part, explain why they reported good OHRQoL. Thus, our findings indicate that a majority of patients tolerate the augmentation and implantation procedures very well and with minor long-term sequelae.

The treatment protocol described in this study, i.e., advanced bone reconstructions under general anesthetics, hospitalization, and sick leave, is considered expensive in a public health services. In the present study, an average of 4.3 days of hospitalization and 20.2 days of sick leave was reported, which is costly for the health service and inconvenient for the patient [33, 43]. When comparing iliac bone graft as a treatment to bone substitutes, a previous study clearly demonstrated that iliac bone graft procedure demands more resources and more than three times the costs of bone substitutes [43]. Although the patients reported good satisfaction and OHRQoL after iliac bone grafting, this treatment is demanding for patients as well as health services, indicating the need for alternative treatment modalities $[37,43,46]$.

\section{Conclusions}

Favorable OHRQoL and satisfaction were reported after advanced reconstruction with iliac crest-derived grafts and implant treatment in orally compromised patients.
However, this treatment requires substantial resources including hospitalization and sick leave.

\section{Supplementary information}

Supplementary information accompanies this paper at https://doi.org/10. 1186/s40729-019-0200-8.

Additional file 1. A self-administered questionnaire.

\section{Abbreviations}

HRQOL: Health-related quality of life; OHIP-14: Oral Health Impact Profile-14; OHRQoL: Oral health-related quality of life; PROMs: Patient-reported outcome measures; QoL: Quality of life

\section{Acknowledgements}

The staff and surgeons at the Department of Oral and Maxillofacial Surgery, Head and Neck Clinic, Haukeland University Hospital, Bergen. Randi Aursland, master student, who helped collecting data. The patients included in this study.

\section{Authors' contributions}

CG contributed to the design of the trial, acquisition of the data, follow-up of the patient-reported data, and analysis and interpretation of the data and drafted the manuscript. SS contributed to the design of the trial, statistical analysis, and analysis and interpretation of the data and drafted the manuscript. EN contributed to the conception and design and critically revised the manuscript. KM contributed to the conception and design and critically revised the manuscript. HG contributed to the design of the trial, statistical analysis, and analysis and interpretation of the data and drafted the manuscript. All authors gave final approval and agreed to be accountable for all aspects of the work.

\section{Funding}

This work was partially funded by the Research Council of Norway through the BEHANDLING project (grant no. 273551) and TROND MOHN Foundation, Norway (BFS2018TMT10).

\section{Availability of data and materials}

The datasets used and/or analyzed during the current study are available from the corresponding author on reasonable request.

\section{Ethics approval and consent to participate}

The Ethics Committee was contacted in 2015, and no ethical approval was needed since this was then considered a quality control study. Written consent was obtained from all participants.

\section{Consent for publication}

Not applicable.

\section{Competing interests}

Cecilie G Gjerde, Siddharth Shanbhag, Evelyn Neppelberg, Kamal Mustafa, and Harald Gjengedal declare that they have no competing interests.

\section{Author details}

${ }^{1}$ Department of Oral and Maxillofacial Surgery, Institute of Clinical Dentistry, University of Bergen, Årstadveien 19, 5009 Bergen, Norway. ${ }^{2}$ Centre for Clinical Dental Research, Institute of Clinical Dentistry, University of Bergen, Bergen, Norway. ${ }^{3}$ Department of Oral and Maxillofacial Surgery, Head and Neck Clinic, Haukeland University Hospital, Bergen, Norway. ${ }^{4}$ Department of Prosthodontics, Institute of Clinical Dentistry, University of Bergen, Bergen, Norway.

Received: 10 October 2019 Accepted: 11 December 2019 Published online: 05 February 2020

References

1. Schaaf H, Lendeckel S, Howaldt HP, Streckbein P. Donor site morbidity after bone harvesting from the anterior iliac crest. Oral Surg Oral Med Oral Pathol Oral Radiol Endod. 2010;109(1):52-8. 
2. Sakkas A, Wilde F, Heufelder M, Winter K, Schramm A. Autogenous bone grafts in oral implantology-is it still a "gold standard"? A consecutive review of 279 patients with 456 clinical procedures. Int J Implant Dent. 2017;3(1):23.

3. Aghaloo TL, Moy PK. Which hard tissue augmentation techniques are the most successful in furnishing bony support for implant placement? Int J Oral Maxillofac Implants. 2007;22(Suppl):49-70.

4. Jensen SS, Terheyden $\mathrm{H}$. Bone augmentation procedures in localized defects in the alveolar ridge: clinical results with different bone grafts and bonesubstitute materials. Int J Oral Maxillofac Implants. 2009;24(Suppl):218-36.

5. Rocchietta I, Fontana F, Simion M. Clinical outcomes of vertical bone augmentation to enable dental implant placement: a systematic review. J Clin Periodontol. 2008;35(8 Suppl):203-15.

6. Kalk WW, Raghoebar GM, Jansma J, Boering G. Morbidity from iliac crest bone harvesting. J Oral Maxillofac Surg. 1996;54(12):1424-9 discussion 30.

7. Seiler JG 3rd, Johnson J. Iliac crest autogenous bone grafting: donor site complications. J South Orthop Assoc. 2000;9(2):91-7.

8. Eufinger $\mathrm{H}$, Leppanen $\mathrm{H}$. lliac crest donor site morbidity following open and closed methods of bone harvest for alveolar cleft osteoplasty. J Craniomaxillofac Surg. 2000;28(1):31-8.

9. Nkenke E, Weisbach V, Winckler E, Kessler P, Schultze-Mosgau S, Wiltfang J, et al. Morbidity of harvesting of bone grafts from the iliac crest for preprosthetic augmentation procedures: a prospective study. Int J Oral Maxillofac Surg. 2004;33(2):157-63.

10. Dawson KH, Egbert MA, Myall RW. Pain following iliac crest bone grafting of alveolar clefts. J Craniomaxillofac Surg. 1996;24(3):151-4.

11. Swan MC, Goodacre TE. Morbidity at the iliac crest donor site following bone grafting of the cleft alveolus. Br J Oral Maxillofac Surg. 2006;44(2):129-33.

12. Jessop ZM, Al-Himdani S, Clement M, Whitaker IS. The challenge for reconstructive surgeons in the twenty-first century: manufacturing tissueengineered solutions. Front Surg. 2015;2:52.

13. Hill NM, Horne JG, Devane PA. Donor site morbidity in the iliac crest bone graft. Aust N Z J Surg. 1999;69(10):726-8.

14. Finkemeier CG. Bone-grafting and bone-graft substitutes. J Bone Joint Surg Am. 2002;84-A(3):454-64.

15. Hernigou P, Desroches A, Queinnec S, Flouzat Lachaniette $\mathrm{CH}$, Poignard A Allain J, et al. Morbidity of graft harvesting versus bone marrow aspiration in cell regenerative therapy. Int Orthop. 2014;38(9):1855-60.

16. Chow A, Mayer EK, Darzi AW, Athanasiou T. Patient-reported outcome measures: the importance of patient satisfaction in surgery. Surgery. 2009;146(3):435-43.

17. Marshall S, Haywood K, Fitzpatrick R. Impact of patient-reported outcome measures on routine practice: a structured review. J Eval Clin Pract. 2006; 12(5):559-68.

18. McGrath C, Lam O, Lang N. An evidence-based review of patient-reported outcome measures in dental implant research among dentate subjects. J Clin Periodontol. 2012;39(Suppl 12):193-201.

19. Helsedepartement DKOo. Oppdragsdokument Helse Vest 2017.

20. Wiklund I. Assessment of patient-reported outcomes in clinical trials: the example of health-related quality of life. Fundam Clin Pharmacol. 2004;18(3):351-63.

21. Newsome PR, McGrath C. Patient-centred measures in dental practice: 1 . An overview. Dent Update. 2006;33(10):596-8 600.

22. Lang NP, Zitzmann NU, Working Group 3 of the VEWoP. Clinical research in implant dentistry: evaluation of implant-supported restorations, aesthetic and patient-reported outcomes. J Clin Periodontol. 2012;39(Suppl 12):133-8.

23. Waldron D, O'Boyle CA, Kearney M, Moriarty M, Carney D. Quality-of-life measurement in advanced cancer: assessing the individual. J Clin Oncol. 1999;17(11):3603-11.

24. Browne JP, O'Boyle CA, McGee HM, Joyce CR, McDonald NJ, O'Malley K, et al. Individual quality of life in the healthy elderly. Qual Life Res. 1994;3(4):235-44.

25. McGrath C, Bedi R. A national study of the importance of oral health to life quality to inform scales of oral health related quality of life. Qual Life Res. 2004;13(4):813-8.

26. John MT, Hujoel P, Miglioretti DL, LeResche L, Koepsell TD, Micheelis W. Dimensions of oral-health-related quality of life. J Dent Res. 2004;83(12):956-60.

27. Slade GD. Derivation and validation of a short-form oral health impact profile. Community Dent Oral Epidemiol. 1997;25(4):284-90.

28. Locker D, Allen F. What do measures of 'oral health-related quality of life' measure? Community Dent Oral Epidemiol. 2007;35(6):401-11.

29. Sischo L, Broder HL. Oral health-related quality of life: what, why, how, and future implications. J Dent Res. 2011;90(11):1264-70.

30. Slade GD. Assessing change in quality of life using the Oral Health Impact Profile. Community Dent Oral Epidemiol. 1998;26(1):52-61.
31. Locker D, Allen PF. Developing short-form measures of oral health-related quality of life. J Public Health Dent. 2002;62(1):13-20.

32. Dahl KE, Wang NJ, Skau I, Ohrn K. Oral health-related quality of life and associated factors in Norwegian adults. Acta Odontol Scand. 2011;69(4):208-14

33. Joshi A, Kostakis GC. An investigation of post-operative morbidity following iliac crest graft harvesting. Br Dent J. 2004;196(3):167-71 discussion 55.

34. Kessler P, Thorwarth M, Bloch-Birkholz A, Nkenke E, Neukam FW. Harvesting of bone from the iliac crest--comparison of the anterior and posterior sites. Br J Oral Maxillofac Surg. 2005;43(1):51-6.

35. Nkenke E, Schultze-Mosgau S, Radespiel-Troger M, Kloss F, Neukam FW. Morbidity of harvesting of chin grafts: a prospective study. Clin Oral Implants Res. 2001;12(5):495-502.

36. Nkenke E, Radespiel-Troger M, Wiltfang J, Schultze-Mosgau S, Winkler G, Neukam FW. Morbidity of harvesting of retromolar bone grafts: a prospective study. Clin Oral Implants Res. 2002;13(5):514-21.

37. Reissmann DR, Dietze B, Vogeler M, Schmelzeisen R, Heydecke G. Impact of donor site for bone graft harvesting for dental implants on health-related and oral health-related quality of life. Clin Oral Implants Res. 2013;24(6):698-705.

38. Vu DD, Schmidt BL. Quality of life evaluation for patients receiving vascularized versus nonvascularized bone graft reconstruction of segmental mandibular defects. J Oral Maxillofac Surg. 2008;66(9):1856-63.

39. Truedsson A, Hjalte K, Sunzel B, Warfvinge G. Maxillary sinus augmentation with iliac autograft - a health-economic analysis. Clin Oral Implants Res. 2013;24(10):1088-93.

40. Papi P, Giardino R, Sassano P, Amodeo G, Pompa G, Cascone P. Oral health related quality of life in cleft lip and palate patients rehabilitated with conventional prostheses or dental implants. J Int Soc Prev Community Dent. 2015;5(6):482-7.

41. Landes CA, Bundgen L, Laudemann K, Ghanaati S, Sader R. Patient satisfaction after prosthetic rehabilitation of bone-grafted alveolar clefts with nonsubmerged ITI Straumann dental implants loaded at three months. Cleft Palate Craniofac J. 2012;49(5):601-8.

42. Reisine S, Freilich M, Ortiz D, Pendrys D, Shafer D, Taxel P. Quality of life improves among post-menopausal women who received bone augmentation during dental implant therapy. Int J Oral Maxillofac Surg. 2012;41(12):1558-62.

43. Dahlin C, Johansson A. Iliac crest autogenous bone graft versus alloplastic graft and guided bone regeneration in the reconstruction of atrophic maxillae: a 5-year retrospective study on cost-effectiveness and clinical outcome. Clin Implant Dent Relat Res. 2011;13(4):305-10.

44. Gjengedal H, Berg E, Gronningsaeter AG, Dahl L, Malde MK, Boe OE, et al. The influence of relining or implant retaining existing mandibular dentures on health-related quality of life: a 2-year randomized study of dissatisfied edentulous patients. Int J Prosthodont. 2013;26(1):68-78.

45. Chiapasco M, Casentini P, Zaniboni M. Bone augmentation procedures in implant dentistry. Int J Oral Maxillofac Implants. 2009;24(Suppl):237-59.

46. Barone A, Ricci M, Mangano F, Covani U. Morbidity associated with iliac crest harvesting in the treatment of maxillary and mandibular atrophies: a 10-year analysis. J Oral Maxillofac Surg. 2011;69(9):2298-304.

47. Hara M, Matsumoto T, Yokoyama S, Higuchi D, Baba K. Location of implantretained fixed dentures affects oral health-related quality of life. Clin Implant Dent Relat Res. 2017;19(4):710-6.

\section{Publisher's Note}

Springer Nature remains neutral with regard to jurisdictional claims in published maps and institutional affiliations.

\section{Submit your manuscript to a SpringerOpen ${ }^{\circ}$ journal and benefit from:}

- Convenient online submission

- Rigorous peer review

- Open access: articles freely available online

High visibility within the field

- Retaining the copyright to your article

Submit your next manuscript at $>$ springeropen.com 